\title{
EQUILIBRIUM STATES OF A BOSE GAS WITH REPULSIVE INTERACTIONS
}

\author{
OLA BRATTELI and DEREK W. ROBINSON
}

(Received 8 February 1980)

\begin{abstract}
We consider infinite volume limit Gibbs states of a nonrelativistic quantum Bose gas consisting of one species of spinless particles with positive interaction potentials. The finite volume reduced density matrices are dominated by the corresponding matrices for the noninteracting gas, and as a consequence all infinite volume limit states are regular, locally normal, and analytic on the appropriate $C C R$ algebra. For sufficiently short range repulsive two-body interactions, the cyclic vector associated with the limit state is separating for the $\sigma$-weak closure of the algebra in the associated representation.
\end{abstract}

\section{Notation and assumptions}

In this paper we study a certain class of non-relativistic quantum Bose gases in thermal equilibrium. We assume that there is only one species of spinless particle, and choose units such that its mass is $\frac{1}{2}$. The appropriate $C^{*}$-algebra is the $C C R$ algebra $\mathfrak{A}$ over $\bigcup_{\Lambda} L^{2}(\Lambda)$, where $\Lambda$ ranges over all open, bounded, subsets in $\mathbf{R}^{v}$, and we have

$$
\mathfrak{A}=\overline{\bigcup_{\Lambda} \mathfrak{A}(\Lambda)},
$$

where $\mathfrak{U}(\Lambda)$ is the $C C R$-algebra over the one particle space $L^{2}(\Lambda)$ (see, for example, [4] for details). In the sequel we consider only bounded regions $\Lambda$ with piecewise smooth boundary $\partial \Lambda$.

The time evolution $\tau^{\wedge}$ of a system of noninteracting particles confined to the region $\Lambda$ is a discontinuous one-parameter group of *-automorphisms of $\mathfrak{U}(\Lambda)$ implemented on Fock space by a unitary group

$$
t \rightarrow \exp \left\{i t d \Gamma\left(T_{\sigma, \Lambda}^{(1)}\right)\right\},
$$

that is, $\tau^{\wedge}$ is the quasifree evolution on $\mathfrak{U}(\Lambda)$ defined by the one-particle evolution

$$
t \rightarrow \exp \left(i t T_{\sigma, \Lambda}^{(1)}\right) \text {. }
$$


Here $\sigma$ is either a smooth, non-negative real function on the boundary $\partial \Lambda$, or $\sigma=+\infty$. In the former case, $T_{\sigma, \Lambda}^{(1)}$ is the self-adjoint extension of the Laplacian $-\nabla^{2}$ corresponding to the boundary condition

$$
\frac{\partial \psi}{\partial n}=\sigma \psi
$$

where $\partial / \partial n$ is the inward normal derivative on $\partial \Lambda$, whilst $T_{\infty, \Lambda}^{(1)}$ is the extension corresponding to Dirichlet boundary conditions, that is, $T_{\infty, \Lambda}^{(1)}$ is the Friedrichs extension of $-\nabla_{\mid c_{0}^{\prime}(\Lambda)}$.

Next, define

$$
T_{\sigma, \Lambda}=d \Gamma\left(T_{\sigma, \Lambda}^{(1)}\right)
$$

as the second quantization of $T_{\sigma, \Lambda}^{(1)}$, and let

$$
T_{\sigma, \Lambda}=\bigoplus_{n=0}^{\infty} T_{\sigma, \Lambda}^{(n)}
$$

be the decomposition of $T_{\sigma . \Lambda}$ corresponding to the direct sum decomposition of the symmetric Fock space $\mathscr{F}(\Lambda)$ into $n$-particle subspaces $L^{2}(\Lambda)_{+}^{n}$, where $L^{2}(\Lambda)_{+}^{n}$ is the subspace of $L^{2}(\Lambda)^{n}=\otimes^{n} L^{2}(\Lambda)$ consisting of functions invariant under permuations of the $n$ arguments in $\Lambda$. Alternatively, $T_{\sigma, \Lambda}^{(n)}$ is the restriction to $L(\Lambda)_{+}^{n}$ of the closure of the operator

$$
\left(T_{\sigma, \Lambda}^{(1)} \otimes \mathbf{I} \otimes \ldots \otimes \mathbf{I}\right)+\left(\mathbf{I} \otimes T_{\sigma, \Lambda}^{(1)} \otimes \ldots \otimes \mathbf{I}\right)+\ldots+\left(\mathbf{I} \otimes \mathbf{I} \otimes \ldots \otimes T_{\sigma, \Lambda}^{(1)}\right)
$$

acting on $L^{2}(\Lambda)^{n}$.

The interaction is defined by an operator $U_{\Lambda}$ of the form

$$
\mathrm{U}_{\Lambda}=\bigoplus_{n=0}^{\infty} \mathrm{U}_{\Lambda}^{(n)} \text {, }
$$

where each $U_{\Lambda}^{(n)}$ is an operator of multiplication,

$$
\left(\mathrm{U}_{\Lambda}^{(n)} \psi\right)\left(x_{1}, \ldots, x_{n}\right)=\mathrm{U}^{(n)}\left(x_{1}, x_{2}, \ldots, x_{n}\right) \psi\left(x_{1}, \ldots, x_{n}\right) .
$$

Here $U^{(n)}$ is a real function, symmetric in the arguments $x_{1}, x_{2}, \ldots, x_{n}$. We assume that each $U^{(n)}$ is positive, that is,

$$
\mathrm{U}^{(n)}\left(x_{1}, \ldots, x_{n}\right) \geqslant 0
$$

for all $x_{1}, \ldots, x_{n} \in \mathbf{R}^{n v}$. In order to define the total Hamiltonian by form sum methods we also assume

$$
\mathrm{U}^{(n)} \in L_{\mathrm{loc}}^{1}\left(\mathbf{R}^{n v} \backslash S_{n}\right)
$$

where $S_{n}$ is a closed set of Lebesque measure zero. This means that $\mathrm{U}^{(n)}$ is integrable over all compact subsets $\mathbf{R}^{n v} \backslash S_{n}$. The requirement that $S_{n}$ has Lebesque measure zero excludes particles with hard cores. As the inclusion of hard cores entails additional 
discussion of the boundary conditions imposed upon the cores, we have made the restriction on $S_{n}$ to avoid too many details, but our results can certainly be extended to this case.

The finite volume $n$-particle Hamiltonian is defined as the form sum, [4], of $T_{\sigma, \Lambda}^{(n)}$ and $U_{\Lambda}$ :

$$
H_{\sigma, \Lambda}^{(n)}=T_{\sigma, \Lambda}^{(n)}+\mathrm{U}_{\Lambda}^{(n)},
$$

and the total finite Hamiltonian is defined by

$$
H_{\sigma, \Lambda}=\bigoplus_{n=0}^{\infty} H_{\sigma, \Lambda}^{(n)},
$$

where $H_{\sigma, \Lambda}^{(0)}=0$.

\section{Functional representation}

Let $p_{\sigma, \Lambda}(x, y ; t)$ be the kernel of the semigroup $t \rightarrow \exp \left(-t T_{\sigma, \Lambda}^{(1)}\right)$, that is, $p_{\sigma, \Lambda}$ is the unique continuous function on $\Lambda \times \Lambda \times \mathbf{R}^{+}$such that the solution of the initial value problem

$$
\begin{aligned}
\frac{\partial \rho}{\partial t} & =\nabla^{2} \rho \text { for } t>0, \quad x \in \Lambda, \\
\frac{\partial \rho}{\partial n}(x, t) & =\sigma(x) \rho(x, t) \quad \text { for } t>0, \quad x \in \partial \Lambda, \\
\rho(x, 0) & =f(x),
\end{aligned}
$$

is given by

$$
\rho(x, t)=\int_{\Lambda} p_{\sigma, \Lambda}(x, y ; t) f(y) d y .
$$

The kernel $p_{o, \Lambda}$ has the properties $[1,2,8]$.

(1) (Positivity conservation)

$$
p_{\sigma, \Lambda}(x, y ; t) \geqslant 0 \text { for } x, y \in \Lambda, \quad t>0 .
$$

(2) (Semigroup property)

$$
\int_{\Lambda} p_{\sigma, \Lambda}(x, y ; t) p_{\sigma, \Lambda}(y, z ; s) d y=p_{\sigma, \Lambda}(x, z ; t+s) .
$$

(3) (Contraction property)

$$
\int_{\Lambda} p_{\sigma, \Lambda}(x, y ; t) d y \leqslant 1
$$

for $x \in \Lambda, t>0$, with equality if and only if $\sigma=0$. 
(4) (Monotonicity in boundary conditions)

If $0 \leqslant \sigma_{1}(x) \leqslant \sigma_{2}(x) \leqslant+\infty$ for all $x \in \partial \Lambda$, then

$$
p_{0, \Lambda}(x, y ; t) \geqslant p_{\sigma_{1}, \Lambda}(x, y ; t) \geqslant p_{\sigma_{2}, \Lambda}(x, y ; t) \geqslant p_{\infty, \Lambda}(x, y ; t)
$$

for all $x, y \in \Lambda, t>0$.

One can construct functional measures corresponding to the transition probabilties $p_{\sigma, A}$ in almost exactly the same manner as Wiener measure is constructed from the infinite volume diffusion process $[1,4]$. The trajectory space can be taken as the set of all functions from the interval $[0, \beta]$ into $\bar{\Lambda}$, that is,

$$
\Omega_{\Lambda, \beta}=\underset{0 \leqslant i \leqslant \beta}{\times} \bar{\Lambda}=(\bar{\Lambda})^{[0, \beta]}
$$

where $\bar{\Lambda}$ is the closure of $\Lambda$. Then $\Omega_{\Lambda, \beta}$ is compact in the product topology. The conditional Wiener measure $\mu_{x, y}^{\sigma, \Lambda, \beta}$ can be defined on $C\left(\Omega_{\Lambda, \beta}\right)$ by specifying it on functions $\phi$ of the form

$$
\phi(\omega)=F\left(\omega\left(t_{1}\right), \ldots, \omega\left(t_{m}\right)\right),
$$

where $F:(\bar{\Lambda})^{m} \rightarrow \mathbf{C}$ is continuous and $0 \leqslant t_{1}<t_{2}<\ldots<t_{m} \leqslant \beta$. One defines

$$
\begin{aligned}
\mu_{x, y}^{\sigma, \Lambda, \beta}(\phi)= & \int_{\Lambda} \ldots \int_{\Lambda} F\left(x_{1}, \ldots, x_{m}\right) p_{\sigma, \Lambda}\left(x, x_{1} ; t_{1}\right) \times p\left(x_{1}, x_{2} ; t_{2}-t_{1}\right) \times \ldots \\
& \times p\left(x_{m-1}, x_{m} ; t_{m}-t_{m-1}\right) \times p\left(x_{m}, y ; \beta-t_{m}\right) d x_{1} \ldots d x_{m} .
\end{aligned}
$$

The Riesz theorem then assures the existence of a unique regular Borel-measure $\mu_{x, y}^{\sigma, \Lambda, \beta}$ on $\Omega_{\Lambda, \beta}$ such that

$$
\mu_{x, y}^{\sigma, \Lambda, \beta}(\phi)=\int_{\Omega_{\Lambda, \xi}} \phi(\omega) d \mu_{x, y}^{\sigma, \Lambda, \beta}(\omega) .
$$

It follows from the monotonicity of $p_{\sigma, \Lambda}$ in $\sigma$ that the functional measures are monotonic in the boundary conditions, that is,

$$
0 \leqslant \sigma_{1} \leqslant \sigma_{2} \leqslant+\infty
$$

implies that

$$
\mu_{x, y}^{\infty, \Lambda, \beta}(B) \leqslant \mu_{x, y}^{\sigma_{2}, \Lambda, \beta}(B) \leqslant \mu_{x, y}^{\sigma_{1}, \Lambda, \beta}(B) \leqslant \mu_{x, y}^{0, \Lambda, \beta}(B)
$$

for all Borel sets $B$.

One can also prove that the measures $\mu_{x, y}^{\sigma, \Lambda, \beta}$ are supported by the set of Hölder continuous trajectories of order $\alpha$ if $0<\alpha<\frac{1}{2}$, and the set of Hölder continuous paths of order $\alpha$ has measure zero if $\frac{1}{2} \leqslant \alpha \leqslant 1$. One can now give a Feynman-Kac type representation of the kernel of the semigroup

$$
\beta \rightarrow \exp \left(-\beta H_{\sigma, \Lambda}^{(1)}\right)
$$


as follows

$$
\exp \left(-\beta H_{\sigma, \Lambda}^{(1)}\right)(x, y)=\int_{\Omega_{\Lambda, \beta}} d \mu_{x, y}^{\sigma, \Lambda, \beta}(\omega) \exp \left\{-\int_{0}^{\beta} d t U^{(1)}(\omega(t))\right\} .
$$

In the case of Dirichlet boundary conditions, $\sigma=+\infty$, this representation simplifies to

$$
\exp \left(-\beta H_{\infty, \Lambda}^{(1)}\right)(x, y)=\int_{\Omega_{s}} d \mu_{x, y}^{\beta}(\omega) \alpha_{\Lambda}(\omega) \exp \left\{-\int_{0}^{\beta} d t \mathrm{U}^{(1)}(\omega(t))\right\},
$$

where $\mu_{x, y}^{\beta}$ is the infinite volume Wiener measure on the trajectory space

$$
\Omega_{\beta}=\left(\dot{\mathbf{R}}^{v}\right)^{[0, \beta]} .
$$

Here $\dot{\mathbf{R}}^{v}$ is the one point compactification of $\mathbf{R}^{v}$, and

$$
\alpha_{\Lambda}(\omega)=\left\{\begin{array}{ll}
1 & \text { if } \omega(t) \in \Lambda \\
0 & \text { otherwise }
\end{array} \text { for all } t \in[0, \beta]\right.
$$

\section{The reduced density matrices}

The positivity assumption on the interaction implies that $\exp \left\{-\beta\left(H_{\sigma, \Lambda}-\mu N_{\Lambda}\right)\right\}$ is of trace class whenever $\beta>0$ and the chemical potential $\mu$ is negative. Here $N_{\Lambda}=d \Gamma\left(\chi_{\Lambda}\right)$ is a local number operator, that is, $N_{\Lambda}$ is the number operator on the symmetric Fock space $\mathscr{F}(\Lambda)$ over $L^{2}(\Lambda)$. We can therefore introduce the finite volume Gibbs states

$$
\omega_{\Lambda}(A)=\operatorname{Tr}_{\mathscr{F}_{(\Lambda)}}\left(e^{-\beta\left(H_{\sigma, \Lambda}-\mu N_{\Lambda}\right)} A\right) / Z_{\Lambda}
$$

for $A \in \mathfrak{Q}(\Lambda)$, where $Z_{\Lambda}=\operatorname{Tr}_{\mathscr{F}(\Lambda)}\left(e^{-\beta\left(H_{\theta, \Lambda}-\mu N_{\Lambda}\right)}\right)$ and $\omega_{\Lambda}, Z_{\Lambda}$ implicitly depend on $\beta, \mu$, $\sigma$ and $U$. The states $\omega_{\wedge}$ and their infinite volume limits are analysed by means of the reduced density matrices

$$
\tilde{\rho}_{\Lambda}\left(y^{m} ; x^{m}\right)=\omega_{\Lambda}\left(a^{*}\left(x_{1}\right) \ldots a^{*}\left(x_{m}\right) a\left(y_{m}\right) \ldots a\left(y_{1}\right)\right),
$$

where $x^{m}=\left(x_{1}, \ldots x_{m}\right), y^{m}=\left(y_{1}, \ldots, y_{m}\right)$ and $a^{*}(x), a(y)$ are the operator valued distributions defining the creation and annihilation operators in the cyclic representation determined by $\omega_{\Lambda}$. Alternatively, $\tilde{\rho}_{\Lambda}\left(x^{m} ; y^{m}\right)$ can be defined as the kernel of the operator $\tilde{\rho}_{\Lambda}$ given by

$$
\tilde{\rho}_{\Lambda}=\sum_{n=0}^{\infty} \frac{(m+n) !}{n !} \operatorname{Tr}_{n}\left(\bar{\rho}_{\Lambda, m+n}\right)
$$

where $\bar{\rho}_{\Lambda, n}$ is the operator

$$
\exp \left\{-\beta\left(H_{\sigma, \Lambda}^{(n)}-\mu n\right)\right\} / Z_{\Lambda}
$$

and $\operatorname{Tr}_{n}$ is the partial trace over $n$ particles. 
Following Ginibre [6] one can now use the Feynman-Kac formula and a combinatorial argument to find an integral representation for $\tilde{\rho}_{\Lambda^{*}}$. The result is, $[4,6]$,

$$
\tilde{\rho}_{\Lambda}\left(x^{m} ; y^{m}\right)=\sum_{\pi} \prod_{i=1}^{m}\left\{\sum_{j_{i}=1}^{\infty} \int d \mu_{x_{i} \pi\left(y_{i}\right)}^{\sigma, \Lambda, j, \beta}\left(\omega_{i}\right)\right\} \rho_{\Lambda}\left(\omega^{m}\right),
$$

where $\omega^{m}=\left(\omega_{1}, \ldots, \omega_{m}\right)$ is a family of $m$ composite trajectories with time intervals $j_{1} \beta, j_{2} \beta, \ldots, j_{m} \beta$, and the first sum runs over all permutations $\pi$ of the points $y_{1}, \ldots, y_{m}$. The correlation functional $\rho_{\mathrm{A}}$ is defined by the formula

$$
\rho_{\Lambda}\left(\omega^{m}\right)=Z_{\Lambda}^{-1} \sum_{n=0}^{\infty} \frac{1}{n !} \int d \tilde{\omega}_{1} \ldots d \tilde{\omega}_{n} z^{q+r} \exp \left\{-U\left(\omega^{m}, \tilde{\omega}^{n}\right)\right\},
$$

where $z=e^{\beta \mu}$ is the activity, $\tilde{\omega}^{n}=\left(\tilde{\omega}_{1}, \ldots, \tilde{\omega}_{n}\right)$ is a family of $n$ composite closed loops and $q$ and $r$ are the number of elementary trajectories that constitute $\omega^{m}$ and $\tilde{\omega}^{n}$ respectively. Moreover, $\mathrm{U}\left(\omega^{m}, \tilde{\omega}^{n}\right)$ is the integral from 0 to $\beta$ of the potential energy of the $(q+r)$ points on the $(m+n)$ trajectories $\omega^{m}, \tilde{\omega}^{n}$ at time $t$ modulo $\beta$, and the integrals $d \tilde{\omega}$ are defined by

$$
d \tilde{\omega}=\sum_{j=1}^{\infty} \frac{1}{j} \int_{\Lambda} d \mu_{u, u}^{\sigma, \Lambda, j \beta}(\tilde{\omega}) d u
$$

The partition function is given by

$$
Z_{\Lambda}=\sum_{n=0}^{\infty} \frac{1}{n !} \int d \tilde{\omega}_{1} \ldots d \tilde{\omega}_{n} z^{r} \exp \left\{-\mathrm{U}\left(\tilde{\omega}^{n}\right)\right\}
$$

In the case of Dirichlet boundary conditions, $\sigma=+\infty$, it is more convenient to redefine $\rho_{\Lambda}$ somewhat, conforming with the conventions in $[4,7]$, that is, one then has

where

$$
\rho_{\Lambda}\left(x^{m} ; y^{m}\right)=\sum_{\pi} \prod_{i=1}^{m}\left\{\sum_{j=1}^{\infty} \int d \mu_{x i, \pi\left(y_{i}\right)}^{j \beta}\left(\omega_{i}\right)\right\} \rho_{\Lambda}\left(\omega^{m}\right)
$$

$$
\begin{aligned}
\rho_{\Lambda}\left(\omega^{m}\right) & =Z_{\Lambda}^{-1} \sum_{n \geqslant 0} \frac{1}{n !} \int d \tilde{\omega}_{1} \ldots d \tilde{\omega}_{n} z^{q+r} \alpha_{\Lambda}\left(\omega^{m}, \tilde{\omega}^{n}\right) \exp \left\{-\mathrm{U}\left(\omega^{m}, \tilde{\omega}^{n}\right)\right\}, \\
d \tilde{\omega} & =\sum_{j=1}^{\infty} \frac{1}{j} \int d \mu_{u, u}^{j \beta}(\tilde{\omega}) d u, \\
Z_{\Lambda} & =\sum_{n=0}^{\infty} \frac{1}{n !} \int d \tilde{\omega}_{1} \ldots d \tilde{\omega}_{n} z^{r} \alpha_{\Lambda}\left(\tilde{\omega}^{n}\right) \exp \left\{-\mathrm{U}\left(\tilde{\omega}^{n}\right)\right\},
\end{aligned}
$$

$d \mu_{x, y}^{t}$ is the infinite volume Wiener measure, and

$$
\alpha_{\Lambda}\left(\omega^{m}, \tilde{\omega}_{n}\right)= \begin{cases}1 & \text { if all the trajectories } \omega_{1}, \ldots, \omega_{m}, \tilde{\omega}_{1}, \ldots, \tilde{\omega}_{n} \\ & \text { remain inside } \Lambda \\ 0 & \text { otherwise. }\end{cases}
$$




\section{Repulsive potentials}

We say that the interaction $U$ is repulsive if

$$
\mathrm{U}^{(m+n)}\left(x^{m}, y^{n}\right) \geqslant \mathrm{U}^{(m)}\left(x^{m}\right)+\mathrm{U}^{(n)}\left(y^{n}\right)
$$

for all $m, n, x^{m} \in \mathbf{R}^{v m}, y^{n} \in \mathbf{R}^{v n}$. Alternatively, this may be characterized by the fact that the $k$-body potential is non-negative for each $k$.

THEOREM 1. Consider a Bose gas with a repulsive interaction $\mathrm{U}$, and let $\tilde{\rho}_{a, \Lambda}^{\mathrm{U}}$ and $\tilde{\rho}_{a, \Lambda}^{0}$ denote the finite volume reduced density matrices with interaction $\mathrm{U}$, and without interaction. It follows that

$$
0 \leqslant \tilde{\rho}_{\sigma, \Lambda}^{\mathrm{U}}\left(x^{m} ; y^{m}\right) \leqslant \tilde{\rho}_{\sigma, \Lambda}^{0}\left(x^{m} ; y^{m}\right) \leqslant \tilde{\rho}_{0, \Lambda}^{0}\left(x^{m} ; y^{m}\right)
$$

for all $x^{m}, y^{m} \in \Lambda^{m}$, all $\beta>0, \mu<0$ and $\sigma \geqslant 0$.

ProOF. It follows from the integral representations in the previous section that it suffices for the two first inequalities to prove that

$$
0 \leqslant \rho_{\sigma, \Lambda}^{\mathrm{U}}\left(\omega^{m}\right) \leqslant \rho_{a, \Lambda}^{0}\left(\omega^{m}\right) .
$$

But the first of these inequalities follows from the representation

$$
\rho_{o, \Lambda}^{U}\left(\omega^{m}\right)=Z_{\Lambda}^{-1} \sum_{n=0}^{\infty} \frac{1}{n !} \int d \tilde{\omega}_{1} \ldots d \tilde{\omega}_{n} z^{q+r} \exp \left\{-\mathrm{U}\left(\omega^{m}, \tilde{\omega}^{n}\right)\right\}
$$

If the interaction is repulsive, one has

$$
\exp \left\{-\mathbf{U}\left(\omega^{m}, \tilde{\omega}^{n}\right)\right\} \leqslant \exp \left\{-\mathbf{U}\left(\omega^{m}\right)\right\} \exp \left\{-\mathbf{U}\left(\tilde{\omega}^{n}\right)\right\}
$$

and therefore

$$
\begin{aligned}
\rho_{a, \Lambda}^{\mathrm{U}}(\omega) & \leqslant z^{q} \exp \left\{-\mathrm{U}\left(\omega^{m}\right)\right\} Z_{\Lambda}^{-1} \times \sum_{n=0}^{\infty} \frac{1}{n !} \int d \tilde{\omega}_{1} \ldots d \tilde{\omega}_{n} z^{r} \exp \left\{-\mathrm{U}\left(\tilde{\omega}^{n}\right)\right\} \\
& =z^{q} \exp \left\{-\mathrm{U}\left(\omega^{m}\right)\right\} \leqslant z^{q} .
\end{aligned}
$$

But the same computation for $U=0$ gives

$$
\rho_{o, \Lambda}^{0}\left(\omega^{m}\right)=z^{q}
$$

and this completes the proof of the second inequality. Finally, the conditional Wiener measures $\mu_{x, y}^{\sigma, \Lambda, t}$ are monotonically decreasing in the boundary conditions $\sigma$, and therefore the largest value of

$$
\tilde{\rho}_{\sigma, \Lambda}^{0}\left(x^{m} ; y^{m}\right)=\sum_{n} \prod_{i=1}^{m}\left\{\sum_{j_{i}=1}^{\infty} d \mu_{x_{i} \pi\left(y_{i}\right)}^{\sigma, \Lambda, j, \beta}\left(\omega_{i}\right)\right\} z^{q}
$$

occurs for the smallest value of $\sigma$, that is, the Neumann conditions $\sigma=0$. 
If one considers Dirichlet boundary conditions one may utilize the monotonicity in $\Lambda$ of the non-interaction reduced density matrices to obtain a bound which is uniform in $\Lambda$ and even valid for $\mu=0$.

THEOREM 2. Consider a Bose gas with a repulsive interaction $\mathrm{U}$, and let $\tilde{\rho}_{\Lambda}^{\mathrm{U}}$ and $\tilde{\rho}_{\Lambda}^{0}$ denote the finite volume reduced density matrices with interaction $\mathrm{U}$, and without interaction, in the presence of Dirichlet boundary conditions. It follows that

and consequently

$$
0 \leqslant \tilde{\rho}_{\Lambda}^{\mathrm{U}}\left(x^{m} ; y^{m}\right) \leqslant \tilde{\rho}_{\Lambda}^{0}\left(x^{m} ; y^{m}\right) \leqslant \lim _{\Lambda \rightarrow \infty} \tilde{\rho}_{\Lambda}^{0}\left(x^{m}, y^{m}\right)
$$

$$
0 \leqslant \tilde{\rho}_{\Lambda}^{\mathrm{U}}\left(x^{m} ; y^{m}\right) \leqslant \sum_{n} \prod_{i=1}^{m} \rho\left(x_{i}-\pi\left(y_{i}\right)\right),
$$

where the last sum is over all permutations of $\left(y_{1}, y_{2}, \ldots, y_{m}\right)$ and

$$
\rho(x)=(2 \pi)^{-v} \int d^{v} p e^{i p x} z e^{-\beta p^{2}}\left(1-z e^{-\beta p^{2}}\right)^{-1} .
$$

Proof. The estimate

$$
0 \leqslant \tilde{\rho}_{\Lambda}^{U}\left(x^{m} ; y^{m}\right) \leqslant \tilde{\rho}_{\Lambda}^{0}\left(x^{m} ; y^{m}\right)
$$

follows from Theorem 1. But

$$
\tilde{\rho}_{\Lambda}^{0}\left(x^{m} ; y^{m}\right)=\sum_{\pi} \prod_{i=1}^{m}\left\{\sum_{j_{1}=1}^{m} d \mu_{x_{i}, \pi\left(y_{i}\right)}^{j_{i} \beta}\left(\omega_{i}\right)\right\} \alpha_{\Lambda}\left(\omega^{m}\right) z^{q}
$$

by the representation in the previous section.

It follows that

$$
\Lambda \rightarrow \tilde{\rho}_{\Lambda}^{0}\left(x^{m} ; y^{m}\right)
$$

is monotonically increasing with limit

where

$$
\sum_{\pi} \prod_{i=1}^{m}\left(\sum_{j=1}^{\infty} z^{j}(4 \pi j \beta)^{-v / 2} e^{-\mid x_{i}-\pi\left(y_{i}||^{2} / 4 j \beta\right.}\right)=\sum_{n} \prod_{i=1}^{m} \rho\left(x_{i}-\pi\left(y_{i}\right)\right)
$$

$$
\rho(x)=\sum_{j=1}^{\infty} z^{j}(4 \pi j \beta)^{-v / 2} e^{-x^{2} / 4 j \beta}=(2 \pi)^{-v} \int d^{v} p e^{i p x} z e^{-\beta p^{2}}\left(1-z e^{-\beta p^{2}}\right)^{-1} .
$$

\section{Thermodynamic limit states for Dirichlet boundary conditions}

If $\mathscr{U}$ is the $C C R$ algebra over $h=\bigcup_{\Lambda} L^{2}(\Lambda)$, and $f \in h$ we let $W(f)$ denote the corresponding Weyl operator, that is, $W(f)$ is unitary and one has

$$
W(f) W(g)=e^{-i I m(f, g) / 2} W(f+g) .
$$


Following Segal (see [4] for details) we say that a state $\omega$ on $\mathfrak{A}$ is regular if

$$
t \rightarrow \omega(W(t f))
$$

is continuous for all $f \in h$ or, equivalently, if

$$
t \rightarrow \pi_{\omega}(W(t f))
$$

is a strongly continuous unitary representation for all $f \in h$, where $\left(\mathscr{H}_{\omega}, \pi_{\omega}, \Omega_{\omega}\right)$ is the cyclic representation associated to $\omega$. If $\omega$ is regular, we define the field operator $\Phi_{\omega}(f)$ as the infinitesimal generator of this unitary operator, and define creation and annihilation operators $a_{\omega}^{*}(f), a_{\omega}(f)$ by

$$
\begin{aligned}
D\left(a_{\omega}(f)\right) & =D\left(a_{\omega}^{*}(f)\right)=D\left(\Phi_{\omega}(f)\right) \bigcap D\left(\Phi_{\omega}(i f)\right) . \\
a_{\omega}(f) & =\left(\Phi_{\omega}(f)+i \Phi_{\omega}(i f)\right) / \sqrt{ } 2,
\end{aligned}
$$

and

$$
a_{\omega}^{*}(f)=\left(\Phi_{\omega}(f)-i \Phi_{\omega}(i f)\right) / \sqrt{ } 2
$$

The state $\omega$ is said to be locally normal if the restriction of $\omega$ to each $\mathfrak{A}(\Lambda)$ is normal with respect to the Fock state. If $\Lambda$ is a bounded region and $\omega$ is a state, define $\hat{N}_{\Lambda}(\omega)=+\infty$ if $\omega$ is not regular on $\mathfrak{A}(\Lambda)$, or if $\Omega_{\omega} \notin D\left(a_{\omega}(f)\right)$ for some $f \in L^{2}(\Lambda)$, and

$$
\hat{N}_{\Lambda}(\omega)=\sup _{\mathscr{F}} \sum_{f \in \mathscr{F}}\left\|a_{\omega}(f) \Omega_{\omega}\right\|^{2}
$$

otherwise, where $\mathscr{F}$ ranges over all finite orthonormal sets in $L^{2}(\Lambda)$. Then $\hat{N}_{\Lambda}$ is an

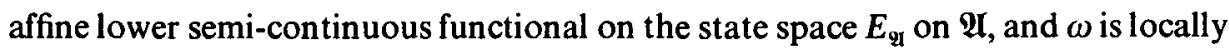
normal if

$$
\hat{N}_{\Lambda}(\omega)<+\infty
$$

for all bounded regions $\Lambda,[4,5]$.

Finally, a state $\omega$ on $\mathfrak{U}$ is said to be analytic if

$$
t \rightarrow \omega(W(t f))
$$

is an analytic function for all $f \in h$ or, equivalently, if $\Omega_{\omega}$ is an analytic vector for $\Phi_{\omega}(f)$ for all $f \in h$.

THEOREM 3. Consider an interacting Bose gas with repulsive interaction, and let $\left\{\omega_{\Lambda}\right\}$ be the net of finite volume Gibbs states for some $\beta>0, \mu<0$ and Dirichlet boundary conditions. Further, let $\left\{\omega_{\Lambda_{\alpha}}\right\}$ be a subnet such that the limits

$$
\omega(A)=\lim _{a} \omega_{\Lambda_{a}}(A)
$$

exist for all $A \in \mathfrak{A}$. 
It follows that $\omega$ has smaller local particle density than the limit Gibbs state $\omega_{0}$ for the non-interacting Bose gas,

$$
\hat{N}_{\Lambda}(\omega) \leqslant \hat{N}_{\Lambda}\left(\omega_{0}\right),
$$

and, in particular, $\omega$ is locally normal. Furthermore, $\omega$ is an entire analytic state and

$$
\lim _{\alpha} \omega_{\Lambda_{z}}\left(\Phi_{\omega \Lambda_{\mathrm{a}}}\left(g_{1}\right) \ldots \Phi_{\omega_{\Lambda_{a}}}\left(g_{n}\right)\right)=\omega\left(\Phi_{\omega}\left(g_{1}\right) \ldots \Phi_{\omega}\left(g_{n}\right)\right)
$$

for all $g_{1}, \ldots, g_{n} \in h$ and all $n \geqslant 1$.

ProOF. By Theorem 2, one has the estimates

$$
0 \leqslant \tilde{\rho}_{\Lambda}^{\mathrm{U}}\left(x^{m} ; y^{m}\right) \leqslant \tilde{\rho}_{\Lambda}^{0}\left(x^{m} ; y^{m}\right) \leqslant \tilde{\rho}\left(x^{m} ; y^{m}\right) \text {, }
$$

where $\tilde{\rho}$ denotes the reduced density matrices for the infinite volume free Gibbs state $\omega_{0}$. Hence one has

$$
\hat{N}_{\Lambda}\left(\omega_{\Lambda^{\prime}}\right)=\int_{\Lambda} d x \tilde{\rho}_{\Lambda^{\prime}}^{U}(x, x) \leqslant \int_{\Lambda} d x \tilde{\rho}(x, x)=\hat{N}_{\Lambda}\left(\omega_{0}\right)
$$

whenever $\Lambda \subset \Lambda^{\prime}$. Since $\hat{N}_{\Lambda}$ is lower semicontinuous, it follows that

$$
\hat{N}_{\Lambda}(\omega) \leqslant \lim _{\alpha} \inf \hat{N}_{\Lambda}\left(\omega_{\Lambda_{\alpha}}\right) \leqslant \hat{N}_{\Lambda}\left(\omega_{0}\right)
$$

and, in particular, $\omega$ is locally normal.

The principal ingredient in the proof of the last part of the theorem is the following real analysis lemma (for a proof see [4]):

LEMMA 1. Let $\left\{f_{\alpha}\right\}$ be a net of $n \geqslant 1$ times continuously differentiable functions from $\mathbf{R}$ into $\mathbf{C}$, and assume that $f_{\alpha}$ converges pointwise to a function $f$. Assume that the derivatives of $f_{\alpha}$ up to order $n$ are bounded on compacts, uniformly in $\alpha$, that is,

$$
\sup _{\alpha} \sup _{t \in[-k, k]}\left|f_{\alpha}^{(m)}(t)\right|<+\infty
$$

for $m=1,2, \ldots, n$, and all $k>0$. It follows that $f$ is $(n-1)$ times continuously differentiable, and

$$
f_{\alpha}^{(m)} \rightarrow f^{(m)}
$$

for $m=0,1, \ldots, n-1$, where the convergence is uniform on compacts.

The proof of Theorem 3 follows from the bounds of Theorem 2 and the entire analyticity of the infinite volume Gibbs state $\omega_{0}$ of the non-interacting Bose gas

$$
\begin{aligned}
\omega_{0}(W(f)) & =\exp \left\{-\omega_{0}\left(\Phi_{\omega_{0}}(f)^{2}\right) / 2\right\} \\
& \left.=\exp \left\{-\left(f,\left(\mathbf{I}+z e^{\beta^{\nabla 2}}\right) / \mathbf{I}-z e^{\beta^{\nabla 2}}\right) f\right) / 4\right\} .
\end{aligned}
$$


First choose $g \in L^{2}(\Lambda)$. Next, note that the states $\omega_{\Lambda}$ and $\omega_{0}$ are all gauge invariant and hence

$$
\omega_{\Lambda}\left(\Phi_{\omega_{\Lambda}}(g)^{n}\right)=0=\omega_{0}\left(\Phi_{\omega_{0}}(g)^{n}\right)
$$

when $n$ is odd. If, however, $n=2 m$ is even,

$$
\begin{aligned}
\omega_{\Lambda}\left(\Phi_{\omega_{\Lambda}}(g)^{2 m}\right) & =\omega_{\Lambda}\left(\left(a_{\omega_{\Lambda}}^{*}(g)+a_{\omega_{\Lambda}}(g)\right)^{2 m}\right) / 2^{m} \\
& =\omega_{\Lambda}\left(a_{\omega_{\Lambda}}^{*}(g)^{2 m}+a_{\omega_{n}}^{*}(g)^{2 m-1} a_{\omega_{\Lambda}}(g)+\ldots\right) / 2^{m}
\end{aligned}
$$

In the last expansion, however, $\omega_{\Lambda}$ vanishes on all terms which contain an unequal number of creation operators $a_{\omega_{\Lambda}}^{*}(g)$ and annihilation operators $a_{\omega_{\Lambda}}(g)$. Thus, by use of the commutation relations

$$
a_{\omega_{\Lambda}}(g) a_{\omega_{\Lambda}}^{*}(g)=a_{\omega_{\Lambda}}^{*}(g) a_{\omega_{\Lambda}}(g)+\|g\|^{2},
$$

one obtains a representation

$$
\begin{aligned}
\omega_{\Lambda}\left(\Phi_{\omega_{\Lambda}}(g)^{2 m}\right) & =\sum_{k=0}^{m} C_{k}^{m} \omega_{\Lambda}\left(a_{\omega_{\Lambda}}^{*}(g)^{k} a_{\omega_{\Lambda}}(g)^{k}\right) \\
& =\sum_{k=0}^{m} C_{k}^{m} \int d x^{k} d y^{k} \tilde{\rho}_{\Lambda}^{\mathrm{U}}\left(x^{k} ; y^{k}\right) \prod_{i=1}^{k} g\left(x_{i}\right) \overline{g\left(y_{i}\right)},
\end{aligned}
$$

where the coefficients are positive polynomials in $\|g\|^{2}$. (We have used $\tilde{\rho}_{\Lambda}^{U}$ to denote the reduced density matrices corresponding to $\omega_{\Lambda}$.) By Theorem 2, however,

$$
0 \leqslant \tilde{\rho}_{\Lambda}^{\mathrm{U}}\left(x^{k} ; y^{k}\right) \leqslant \tilde{\rho}\left(x^{k} ; y^{k}\right),
$$

where $\tilde{\rho}$ are the reduced density matrices associated with the infinite volume noninteracting equilibrium state $\omega_{0}$. Thus, since $C_{k}^{m} \geqslant 0$, one has

$$
\begin{aligned}
0 \leqslant \omega_{\Lambda}\left(\Phi_{\omega_{\Lambda}}(g)^{2 m}\right) & \leqslant \sum_{k=0}^{m} C_{k}^{m} \int d x^{k} d y^{k} \tilde{\rho}^{0}\left(x^{k} ; y^{k}\right) \prod_{i=1}^{k}\left|g\left(x_{i}\right)\right|\left|\overline{g\left(y_{i}\right)}\right| \\
& =\omega^{0}\left(\Phi_{\omega_{0}}(|g|)^{2 m}\right) .
\end{aligned}
$$

The last evaluation follows from the gauge-invariance of $\omega^{0}$ and the definition of the $C_{k}^{m}$.

Next introduce the family of functions $f_{a}: \mathbf{R} \rightarrow \mathbf{C}$

$$
f_{a}(t)=\omega_{\Lambda_{a}}(W(t g)) \text {. }
$$

These functions converge pointwise to the function $f: \mathbf{R} \rightarrow \mathbf{C}$ defined by

$$
f(t)=\omega(W(t g)) .
$$


Furthermore, they satisfy the hypotheses of Lemma 1 for all $n$. For example,

$$
\begin{aligned}
\left|f_{a}^{(m)}(t)\right|^{2} & =\left|i^{m} \omega_{\Lambda_{a}}\left(\Phi_{\omega_{\Lambda_{a}}}(g)^{m} W(t f)\right)\right|^{2} \\
& \leqslant\left|\omega_{\Lambda_{a}}\left(\Phi_{\omega_{\Lambda_{a}}}(g)^{2 m}\right)\right| \\
& \leqslant \omega^{0}\left(\Phi_{\omega^{0}}(|g|)^{2 m}\right) .
\end{aligned}
$$

Hence $f \in C^{\infty}(\mathbf{R})$ and $f_{a}^{(m)} \rightarrow f^{(m)}$ uniformly on compacts. But, if $\bar{F}_{0}: \mathbf{R} \rightarrow \mathbf{C}$ is defined by

$$
F_{0}(t)=\omega^{0}(W(t|g|))
$$

then $F_{0}$ is entire analytic and

$$
\begin{aligned}
\left|f^{(m)}(0)\right| & =\lim _{\alpha}\left|f_{\alpha}^{(m)}(0)\right| \\
& \leqslant \omega^{0}\left(\Phi_{\omega_{0}}(|g|)^{m}\right) \\
& =\left|F_{0}^{(m)}(0)\right| .
\end{aligned}
$$

Thus $f$ is an entire analytic function. Therefore

$$
t \rightarrow \omega(W(t g))
$$

is an entire analytic function for all $g \in L^{2}\left(\mathbf{R}^{v}\right)$ with compact support and hence $\omega$ is an entire analytic state.

Next, from convergence of the derivatives, one has

$$
\begin{aligned}
\omega_{\Lambda_{a}}\left(\Phi_{\omega_{\Lambda_{\alpha}}}(g)^{n}\right) & =i^{-n} f_{a}^{(n)}(0) \\
& \rightarrow i^{-n} f^{(n)}(0)=\omega\left(\Phi_{\omega}(g)^{n}\right) .
\end{aligned}
$$

The more general statement of the theorem with $n$ different functions $g_{1}, \ldots, g_{n}$ is obtained in a similar manner by examining derivatives of a function $f_{\alpha}\left(t_{1}, \ldots, t_{n}\right)=$ $\omega_{\Lambda_{x}}\left(W\left(t_{1} g_{1}+t_{2} g_{2}+\ldots t_{n} g_{n}\right)\right)$ of several variables. We omit the details.

There is a partial converse of Theorem 3.

COROLlary 1. Adopt the assumptions of Theorem 3 but further assume that the reduced density matrices $\tilde{\rho}_{\Lambda}^{U}$ associated with the Gibbs states $\omega_{\Lambda}$ are pointwise convergent. It follows that the states are weakly* convergent.

Proof. It follows from the bounds

$$
0 \leqslant \tilde{\rho}_{\Lambda}^{U}\left(x^{m} ; y^{m}\right) \leqslant \tilde{\rho}\left(x^{m} ; y^{m}\right) .
$$

and the Lebesgue dominated convergence theorem that $\omega_{\Lambda}\left(\Phi_{\omega_{\Lambda}}(g)^{2 m}\right)$ converges for 
all $g \in L^{2}\left(\mathbf{R}^{v}\right)$ with compact support. Moreover, the estimates

$$
0 \leqslant \omega_{\Lambda}\left(\Phi_{\omega_{\Lambda}}(g)^{2 m}\right) \leqslant \omega^{0}\left(\Phi_{\omega^{0}}(|g|)^{2 m}\right)
$$

imply that one can define numbers

$$
\omega(W(g))=\sum_{m=0}^{\infty} \frac{(-1)^{m}}{(2 m) !} \lim _{\Lambda \rightarrow \infty} \omega_{\Lambda}\left(\Phi_{\omega_{\Lambda}}(g)^{2 m}\right)
$$

for each such $g$. It is then readily verified that these numbers uniquely determine an entire analytic state $\omega$ over the $C C R$ algebra and $\omega_{\Lambda} \rightarrow \omega$.

Theorem 3 states properties of the thermodynamic limit with $\beta>0$ and $\mu<0$ fixed. It is also possible to consider the thermodynamic limit with $\beta>0$ and the overall particle density fixed. (This latter form of limit is essential for the discussion of Bose-Einstein condensation of the ideal Bose gas.) The basic difficulty for the ideal gas with Dirichlet boundary conditions is that the formalism is only defined for $z=\exp \{\beta \mu\} \leqslant 1$ and for $z$ fixed in this range the local particle densities $\rho_{\Lambda}(\beta, z)$ are uniformly bounded, whenever $v \geqslant 3$, by the density of the infinite ideal gas at $z=1$, that is,

$$
\rho_{\Lambda}(\beta, z) \leqslant \rho_{c}(\beta)=(2 \pi)^{-v} \int d^{v} p\left(e^{\beta p^{2}}-1\right)^{-1} .
$$

Now it follows from the bound $\hat{N}_{\Lambda}(\omega) \leqslant \hat{N}_{\Lambda}\left(\omega^{0}\right)$ that the density of the interacting gas is also uniformly bounded by $\rho_{c}(\beta)$ if $z \leqslant 1$. This seeming paradox is resolved for the ideal gas by remarking that if $\rho_{\Lambda}(\beta, z)>\rho_{c}(\beta)$ one must have $z>1$ for each finite $\Lambda$ and in the thermodynamic limit at fixed density this leads to a range of choices $z_{\Lambda}$ such that $z_{\Lambda} \rightarrow 1$. The discussion of the interacting system at fixed density is less tractable and it is not evident that the corresponding $z_{\Lambda}$ would converge to a critical finite value. Nevertheless, the above method of bounds could be used to discuss properties of any limit for which $z_{\Lambda} \rightarrow 1$.

\section{Thermodynamic limit states for general boundary conditions}

We will now prove results corresponding to those in Section 5 for more general boundary conditions than the Dirichlet one. The difficulty which occurs if, for example, one uses Neumann boundary conditions is that the density matrices for rectangular regions $\Lambda$ (and conceivably general convex regions) dominate the infinite density matrices, and this leads to the estimates

$$
\hat{N}_{\Lambda}\left(\omega_{\Lambda^{\prime}}\right) \geqslant \hat{N}_{\Lambda}\left(\omega_{0}\right) \text {, }
$$

which are converse to the corresponding estimates for Dirichlet boundary condition. The situation, to some extent, can be remedied by the following lemma. 
LEMMA 2. Let $R>0$ be a constant. Then there exists a constant $M=M(R, \beta)$ such that

$$
\tilde{\rho}_{\sigma, \Lambda}^{0}\left(x^{m} ; y^{m}\right) \leqslant m !\left(\frac{z M}{1-z}\right)^{m}
$$

for all $x^{m}, y^{m} \in \Lambda^{m}$, all $m \geqslant 1$ and all bounded regions $\Lambda$ satisfying one of the following two requirements:

1. The boundary $\partial \Lambda$ is a $C^{2}$-surface of mean curvature less then $(1 / R)$.

2. $\Lambda$ is a rectangular region whose sides are longer than $R$.

Proof. One has

$$
\tilde{\rho}_{0, \Lambda}^{0}\left(x^{m} ; y^{m}\right)=\sum_{\pi} \prod_{j=1}^{m} \tilde{\rho}_{0, \Lambda}^{0}\left(x_{j}, \pi\left(y_{j}\right)\right),
$$

where $\pi$ runs over all permutations of $y_{1}, \ldots, y_{m}$, and

$$
\tilde{\rho}_{0, \Lambda}^{0}(x ; y)=\sum_{j=1}^{\infty} z^{j} p_{0, \Lambda}(x, y ; j \beta) .
$$

Thus the bound of the lemma will follow once one can establish the bound

$$
\left|p_{0 . \Lambda}(x, y ; t)\right| \leqslant M(R, \beta)
$$

when $t \geqslant \beta$. In case 1 these bounds are an immediate consequence of Proposition A3 in [2], and in case 2 they follow from the explicit expression for $p_{0, \Lambda}$ in $[2,7]$.

THEOREM 4. Consider an interacting Bose gas with repulsive interaction, and let $\left\{\omega_{\Lambda}\right\}$ be the net of finite volume Gibbs states for some $\beta>0, \mu<0$ and general $\sigma$ boundary conditions. Further, let $\left\{\omega_{\Lambda_{\alpha}}\right\}$ be a subnet such that $\Lambda_{\alpha} \rightarrow \mathbf{R}^{v}$ and the limits

$$
\omega(A)=\lim _{\alpha} \omega_{\Lambda_{a}}(A)
$$

exist for all $A \in \mathfrak{A}$, and each $\Lambda_{\alpha}$ is either a convex region with a $C^{3}$ boundary $\partial \Lambda$ with mean curvature bounded by an $\alpha$-independent number, or a rectangular region.

It follows that

$$
\hat{N}_{\Lambda}(\omega)<+\infty
$$

for all bounded regions $\Lambda$ and, in particular, $\omega$ is locally normal. Furthermore, $\omega$ is an entire analytic state and

$$
\lim _{\alpha} \omega_{\Lambda_{\alpha}}\left(\Phi_{\omega_{\Lambda_{a}}}\left(g_{1}\right) \ldots \Phi_{\omega_{\Lambda_{\alpha}}}\left(g_{n}\right)\right)=\omega\left(\Phi_{\omega}\left(g_{1}\right) \ldots \Phi_{\omega}\left(g_{n}\right)\right)
$$

for all $g_{1}, \ldots, g_{n} \in h$ and all $n \geqslant 1$.

Proof. Theorem 1 and Lemma 2 imply that

$$
\begin{aligned}
0 \leqslant \rho_{\sigma, \Lambda_{a}}^{U}\left(x^{m} ; y^{m}\right) & \leqslant \tilde{\rho}_{0, \Lambda}^{0}\left(x^{m}, y^{m}\right) \\
& \leqslant m !\left(\frac{z M}{1-z}\right)^{m}
\end{aligned}
$$


for all sufficiently large $\alpha$. In particular,

$$
\hat{N}_{\Lambda}\left(\omega_{\Lambda_{z}}\right)=\int_{\Lambda} d x \tilde{\rho}_{\sigma, \Lambda_{a}}(x, x) \leqslant \frac{z M}{1-z}|\Lambda|
$$

where $|\Lambda|$ is the volume of $\Lambda$. Since $\hat{N}_{\Lambda}$ is lower semicontinuous, it follows that

$$
\hat{N}_{\Lambda}(\omega) \leqslant \frac{z M}{1-z}|\Lambda|<+\infty
$$

and $\omega$ is locally normal.

We compute as in the proof of Theorem 3 that, if $g \in L^{2}(\Lambda)$ and $\Lambda \subseteq \Lambda_{a}$, then

$$
\omega_{\Lambda_{g}}\left(\Phi_{\omega_{\Lambda_{a}}}(g)^{2 m}\right)=\sum_{k=0}^{m} C_{k}^{m} \omega_{\Lambda_{a}}\left(a_{\omega_{\Lambda_{g}}}^{*}(g)^{k} a_{\omega_{\Lambda_{z}}}(g)^{k}\right)
$$

and a combinatorial argument establishes that

$$
C_{k}^{m}=\frac{(2 m) !}{(k !)^{2}(m-k) ! 2^{2 m-k}}\|g\|^{2(m-k)} .
$$

From the estimate in the beginning of the proof one obtains

$$
\begin{aligned}
\omega_{\Lambda_{a}}\left(a_{\omega_{\Lambda_{a}}}^{*}(g)^{k} a_{\omega_{\Lambda_{a}}}(g)^{k}\right) & =\int_{\Lambda^{k}} d x^{k} \int_{\Lambda^{k}} d y^{k} \tilde{\rho}_{a, \Lambda_{a}}^{\mathrm{U}}\left(y^{k} ; x^{k}\right) \prod_{i=1}^{k} g\left(x_{i}\right) \overline{g\left(y_{i}\right)} \\
& \left.\leqslant\|g\|^{2 k}\left[\int_{\Lambda^{k}} d x^{k} \int_{\Lambda^{k}} d y^{k} \tilde{\rho}_{\sigma, \Lambda_{a}}^{U} y^{k}, x^{k}\right)^{2}\right]^{\frac{1}{t}} \\
& \leqslant\|g\|^{2 k}|\Lambda|^{k} k !\left(\frac{z M}{1-z}\right)^{k}
\end{aligned}
$$

Therefore, if $\lambda>0$, one has

$$
\begin{aligned}
\sum_{m \geqslant 0} & \frac{\lambda^{m}}{m !}\left|\omega_{\Lambda_{a}}\left(\Phi_{\omega_{\Lambda_{a}}}(g)^{m}\right)\right| \\
& =\sum_{m \geqslant 0} \frac{\lambda^{2 m}}{(2 m) !} \omega_{\Lambda_{a}}\left(\Phi_{\omega_{\Lambda_{a}}}(g)^{2 m}\right) \\
& \leqslant \sum_{m \geqslant 0} \sum_{k=0}^{m} \frac{\lambda^{2 m}(2 m) !|\Lambda|^{k} k ![z M /(1-z)]^{k}}{(2 m) !(k !)^{2}(m-k) ! 2^{2 m-k}}\|g\|^{2 m} \\
& =\sum_{k=0}^{\infty}\left(\frac{\lfloor\Lambda \mid z M}{2}\right)^{k} / k ! \sum_{m=k}^{\infty}\left(\frac{\lambda\|g\|}{2}\right)^{2 m} /(m-k) ! \\
& =\exp \left\{\left(\frac{\lambda\|g\|}{2}\right)^{2}\right\}_{k=0}^{\infty}\left(\frac{|\Lambda| z M \lambda^{2}\|g\|^{2}}{8(1-z)}\right)^{k} / k ! \\
& =\exp \left\{\left(\frac{\lambda\|g\|}{2}\right)^{2}\left(1+\frac{|\Lambda| z M}{2(1-z)}\right)\right\}<+\infty .
\end{aligned}
$$


Since the last bound is independent of $\alpha$ one can now use Lemma 1 as in the proof on Theorem 3 to establish the last statement of Theorem 4.

One can similarly prove the analogue of Corollary 1 in this setting.

COROLLARY 2. Let $\mathrm{U}$ be a repulsive interaction and let $\Lambda_{a}$ be a net of bounded regions of the type described in Theorem 7 such that the reduced density matrices $\tilde{\rho}_{\sigma, \Lambda_{s}}^{\mathrm{U}}$ are pointwise convergent as $\alpha \rightarrow \infty$. It follows that the associated Gibbs state $\omega_{\Lambda_{a}}$ are weakly* convergent as $\alpha \rightarrow \infty$.

\section{Modular structure}

We now consider interactions defined by translationally invariant two-body potentials $\Psi$, that is,

$$
\mathrm{U}^{(n)}\left(x_{1}, \ldots, x_{n}\right)=\sum_{1 \leqslant i<j \leqslant n} \Psi\left(x_{i}-x_{j}\right)
$$

and

$$
\mathrm{U}=\frac{1}{2} \iint d x d y \Psi(x-y) a^{*}(x) a^{*}(y) a(y) a(x)
$$

Theorem 5. Consider the finite volume $\sigma$-boundary conditions Gibbs states $\omega_{\Lambda}$ at temperature $\beta>0$ and chemical potential $\mu<0$ for a Bose gas interacting through a two-body potential $\Psi$ satisfying

1. $\Psi$ is positive, that is,

$$
\Psi(x) \geqslant 0 \text { for all } x \in \mathbf{R}^{v},
$$

and

2. The estimate

$$
\int d^{v} x\left(1+|x|^{2}\right)^{v+\varepsilon}|\Psi(x)|^{2}<+\infty
$$

is valid for some $\varepsilon>0$.

Let $\Lambda_{\alpha}$ be a net of bounded regions such that $\Lambda_{\alpha} \rightarrow \mathbf{R}^{v}$ and such that $\Lambda_{\alpha}$ satisfies the requirements in Theorem 4 in the case of non-Dirichlet boundary conditions, and assume that $\omega=\lim _{\alpha} \omega_{\Lambda_{a}}$ exists in the weak* topology.

It follows that $\omega$ is a modular state, that is, the cylic vector $\Omega_{\omega}$ in the representation $\left(\mathscr{H}_{\omega}, \pi_{\omega}, \Omega_{\omega}\right)$ determined by $\omega$ is separating for $\pi_{\omega}(\mathfrak{(})^{\prime \prime}$.

Proof. We prove the theorem via two lemmas. 
LEMMA 3. The estimate

$$
\omega_{\Lambda_{z}}\left(\left(N_{\Lambda}+1\right)^{m}\right) \leqslant K(m)(|\Lambda|+1)^{m}<+\infty
$$

is valid for all bounded regions $\Lambda \subseteq \Lambda_{\alpha}$ and all $m \geqslant 0$, where $\omega_{\Lambda_{a}}\left(N_{\Lambda}^{m}\right)$ is defined by the normal extension of $\omega_{\Lambda_{a}}$ from $\mathfrak{U}(\Lambda)$ to $\mathfrak{U}(\Lambda)^{\prime \prime}$ as in [3], and $K(m)$ is independent of $\alpha$ and $\Lambda$.

Proof. One has

$$
\begin{aligned}
& \omega_{\Lambda_{a}}\left(N_{\Lambda}\left(N_{\Lambda}-1\right) \ldots\left(N_{\Lambda}-k+1\right)\right) \\
& \quad=\int_{\Lambda^{k}} d x^{k} \omega_{\Lambda_{a}}\left(a_{\omega_{\Lambda_{a}}}^{*}\left(x_{1}\right) \ldots a_{\omega_{\Lambda_{a}}}^{*}\left(x_{k}\right) \ldots a_{\omega_{\Lambda_{a}}}^{*}\left(x_{k}\right) a_{\omega_{\Lambda_{a}}}\left(x_{1}\right)\right) \\
& \quad=\int_{\Lambda^{k}} d x^{k} \tilde{\rho}_{\Lambda_{a}}\left(x^{k} ; x^{k}\right) \\
& \quad \leqslant \int_{\Lambda^{k}} d x^{k} \tilde{\rho}_{0, \Lambda_{a}}^{0}\left(x^{k} ; x^{k}\right) \\
& \leqslant k !\left(\frac{z M}{1-z}\right)^{k}|\Lambda|^{k}<+\infty
\end{aligned}
$$

The last two estimates follow from Theorem 1 and Lemma 2. The coefficients $S_{k}^{m}$ in the expansion

$$
\rho^{m}=\sum_{k=1}^{m} S_{k}^{m} \rho(\rho-1) \ldots(\rho-k+1)
$$

are positive by the recursion relation

$$
S_{k}^{m+1}=S_{k-1}^{m}+k S_{k}^{m}, \quad S_{m}^{m}=S_{1}^{m}=1,
$$

and hence

$$
N_{\Lambda}^{m}=\sum_{k=1}^{m} S_{k}^{m} N_{\Lambda}\left(N_{\Lambda}-1\right) \ldots(N-k+1)
$$

is a linear combination of positive operators on $D\left(N_{\Lambda}^{m}\right)$. Therefore

$$
\omega_{\Lambda_{a}}\left(N_{\Lambda}^{m}\right) \leqslant \sum_{k=1}^{m} S_{k}^{m} k !\left(\frac{z M}{1-2}\right)^{k}|\Lambda|^{k}<+\infty
$$

uniformly is $\Lambda_{\alpha}$. The estimate in the lemma is an immediate consequence of this estimate.

LEMMA 4. Let $f \in C^{2}$ be a function with support contained in a bounded open region $\Lambda$; let $\Psi$ be a two-body interaction such that

$$
\|\mid \Psi\|_{e}=\left\{\int d^{v} x\left(1+|x|^{2}\right)^{v+\varepsilon}|\Psi(x)|^{2}\right\}^{\frac{1}{2}}<+\infty
$$


for some $\varepsilon>0$, and let

$$
\mathrm{U}_{\Lambda}=\frac{1}{2} \int_{\Lambda} \int_{\Lambda} d x d y \Psi(x-y) a^{*}(x) a^{*}(y) a(y) a(x) .
$$

It follows that

$$
\left\|\left[T_{\sigma, \Lambda}, W(f)\right] \psi\right\| \leqslant\|\nabla f\|_{2}^{2}\|\psi\|+2\left\|\nabla^{2} f\right\|{ }_{2}\left\|\left(N_{\Lambda}+I\right)^{\frac{1}{2}} \dot{\psi}\right\|
$$

for $\psi \in D\left(N_{\AA}^{\ddagger}\right)$

$$
\left\|\left[\mathrm{U}_{\Lambda}, W(f)\right] \psi\right\| \leqslant d\left(K_{\psi}, f, \varepsilon\right)\|\Psi\|_{\varepsilon}
$$

if $\psi \in D\left(N_{\Lambda^{\prime}}^{3 / 2}\right)$ and

$$
\left\|\left(N_{\Lambda^{\prime}}+1\right)^{3 / 2} \psi\right\| \leqslant K_{\psi}\left(\left|\Lambda^{\prime}\right|+1\right)^{3 / 2}
$$

for all bounded regions $\Lambda^{\prime}$, where $K_{\psi}$ is independent of $\Lambda^{\prime}$ and the constant $d$ is independent of $\Lambda^{\prime}$, and finally

$$
\left\|\left[N_{\Lambda}, W(f)\right] \psi\right\| \leqslant b(f)\left\|\left(N_{\Lambda}+1\right)^{\frac{1}{2}} \psi\right\|
$$

for all $\psi \in D\left(N_{\bar{\Lambda}}^{\mathrm{t}}\right)$, and $b$ is independent of $\Lambda$.

Proof. The estimates on the commutators by $T_{\sigma, \Lambda}$ and $U_{\Lambda}$ follow from the appendix of [3]. The last estimate is clear from the formula

$$
\left[N_{\Lambda}, W(f)\right]=W(f)\left\{\phi(i f)+\frac{1}{2}\|f\|^{2} 1\right\} .
$$

END OF PROOF OF THEOREM 5. Let $\mathscr{D}_{\Lambda}$ be the *-algebra generated algebraically by the operators of the form $W(f)$, where $f$ is a $C^{2}$-function with compact support inside $\Lambda$. Since all elements in $\mathscr{D}_{\Lambda}$ are finite linear combinations of $W(f)$ 's it follows from Lemmas 3 and 4 that

$$
\omega_{\Lambda_{g}}\left(\left[K_{\Lambda_{z}}, A\right]^{*}\left[K_{\Lambda_{g}}, A\right]\right) \leqslant C(A)
$$

for all $A \in \mathscr{D}_{\Lambda}$ and all $\Lambda_{\alpha} \supseteq \Lambda$ where the constant $C(A)$ is independent of $\alpha$, and

$$
K_{\Lambda_{z}}=H_{\sigma, \Lambda_{a}}-\mu N_{\Lambda_{a}}
$$

The theorem now follows from [3], Theorem 1 (see also [9]) and the local normality of $\omega$.

\section{References}

[1] N. Angelescu and G. Nenciu, "On the independence of the thermodynamic limit on the boundary conditions in quantum statistical mechanics", Commun. Math. Phys. 29 (1973), 15-30. 
[2] R. Arima, "On general boundary value problems for parabolic equations", J. Math. Kyoto University 4 (1964), 207-243.

[3] O. Bratteli and D. W. Robinson, "Green's functions, Hamiltonians, and modular automorphisms", Commun. Math. Phys. 50 (1976), 133-156.

[4] O. Bratteli and D. W. Robinson, Operator algebras and quantum statistical mechanics, Vol. 2 (Springer-Verlag, 1980).

[5] M. Courbage, S. Miracle-Sole and D. W. Robinson, "Normal states and representations of the canonical communication relations", Ann. Inst. Henri Poincaré 14A (1971), 171-178.

[6] J. Ginibre, "Reduced density matrices for quantum gases I. Limit of infinite volume", J. Math. Phys. 6 (1965), 238-251.

[7] J. Ginibre, "Some applications of functional integration in statistical mechanics" in C. de Witt and R. Stora (eds.), Statistical mechanics and field theory (Gordon and Breach, New York, 1971).

[8] S. Ito, "Fundamental solutions of parabolic differential equations and boundary value problems", Jap. J. Math 27 (1975), 55-102.

[9] M. Winnink, "Some general properties of equilibrium states in an algebraic approach", in R. Sen and C. Weil (eds.), Statistical mechanics and field theory (Keter Publishing House, Jerusalem, 1971).

Institute of Mathematics

NTH, Trondheim 7034

Norway

and

School of Mathematics

University of New South Wales

Kensington

NSW 2033 